\title{
Hyers-Ulam-Rassias Stability of Quadratic Functional Equations in 2-Banach Spaces
}

\author{
Manoj Kumar ${ }^{1}$, Renu Chugh ${ }^{2}$, Ashish $^{3}$ \\ ${ }^{1}$ Assistant Professor, ${ }^{2}$ Professor, ${ }^{3}$ Research Scholar \\ Department of Mathematics \\ Maharshi Dayanand University, Rohtak \\ 124001, INDIA
}

\begin{abstract}
In this paper, using the direct method we study the generalized Hyers-Ulam-Rassias stability of the following quadratic functional equations $f(2 x \pm y)=f(x \pm y)+6 f(x)$ and $f(3 x \pm y)=f(x \pm y)+16 f(x)$ for the mapping $f$ from normed linear space in to 2-Banach spaces.
\end{abstract}

Keywords: Linear 2-normed space, 2-Banach spaces, Quadratic functional equations, Stability.

\section{INTRODUCTION}

In 1940, S. M. Ulam [10], triggered the study of stability problems for various functional equations. He suggested the stability problem of functional equations concerning the stability of group homomorphism. In the next year, D. H. Hyers [3] was the first mathematician to present the result concerning the stability of functional equations on Banach spaces. The generalized version of D. H. Hyers [3] result was given by famous Greece mathematician Th. M. Rassias [11] in 1978. The stability paper [12] given by Th. M. Rassias has significantly influenced in the development of stability of functional equations. Further, in 1994, P. Gavruta [6] provided a further generalization in which he replaced the bound $\theta\left(\|x\|^{p}+\|y\|^{p}\right)$ by a general function $\phi(x, y)$ for the existence of unique linear mapping. The functional equation $f(2 x+y)+f(2 x-y)=f(x+y)+f(x-y)+6 f(x)(1.1)$ is called as quadratic functional equation. Since the function $f(x)=c x^{2}$ is a solution of the above quadratic functional equation (1.1). In 2002, I. S. Chang and H. M. Kim [5] established the general solution and proved the generalized Hyers-Ulam-Rassias stability of the quadratic functional equation (1.1). The functional equation

$f(3 x+y)+f(3 x-y)=f(x+y)+f(x-y)+16 f(x)(1.2)$ is also called as quadratic functional equation. In 2011, H. A. Kenary et.al. [4] proved the generalized Hyers-Ulam-Rassias stability of the quadratic functional equation (1.2) using fixed point method. Recently, W. G. Park [13] investigated the stability of approximate additive mappings, approximate Jensen mappings and approximate quadratic mappings in 2Banach spaces.

In section 1, we adopt some usual terminology, notations and conventions which will be used later in the next sections. In Section 2 we establish the Hyers-Ulam-Rassias stability of the quadratic functional equation (1.1) in 2-Banach space. Further in the last section we established the Hyers-Ulam-Rassias stability of the quadratic functional equation (1.2) in 2-Banach space. In 1960s, S. Gahler [8, 9] introduced the concept of linear 2-normed spaces and established the following definition:
Definition 1.1. Let $A$ be a linear space over $\mathfrak{R}$ with $\operatorname{dim} A>1$ and let $\|.,\|:. A \times A \rightarrow \Re$ be a function satisfying the following properties:

(a) $\|x, y\|=0$ if and only if $x$ and $y$ are linearly dependent,

(b) $\|x, y\|=\|y, x\|$,

(c) $\|\lambda x, y\|=|\lambda|\|x, y\|$,

(d) $\|x, y+z\| \leq\|x, y\|+\|x, z\|$,

for all $x, y, z \in A$ and $\lambda \in \mathfrak{R}$. Then the function $\|, .$,$\| is called$ a 2-norm on $A$ and the pair $(A,\|.\|$,$) is called a linear 2-$ normed space. Sometimes the condition (d) called the triangle inequality.

In 2011, W. G. Park [13] introduces a basic property of linear 2-normed spaces as follows.

Lemma 1.2. Let $(A,\|, .\|$,$) be a linear 2-normed space. If$ $\|x, y\|=0$ for all $y \in A$, then $x=0$.

In the 1960 's, S. Gahler and A. White $[1,2,7,8]$ also introduced the concept of 2-Banach spaces. In order to define completeness, the concepts of Cauchy sequences and convergence are required.

Definition 1.3. A sequence $\left\{x_{n}\right\}$ in a linear 2-normed space $A$ is called a Cauchy sequence if there are two points $y, z \in A$ such that $y$ and $z$ are linearly independent, $\lim _{l, m \rightarrow \infty}\left\|x_{l}-x_{m}, y\right\|=0$ and $\lim _{l, m \rightarrow \infty}\left\|x_{l}-x_{m}, z\right\|=0$.

Definition 1.4. A sequence $\left\{x_{n}\right\}$ in a linear 2-normed space $A$ is called a convergent sequence if there is an $x \in A$ such that $\lim _{n \rightarrow \infty}\left\|x_{n}-x, y\right\|=0$ for all $y \in A$. If $\left\{x_{n}\right\}$ converges to $x$, write $x_{n} \rightarrow x$ as $n \rightarrow \infty$ and call $x$ the limit of $\left\{x_{n}\right\}$. In this case, we also write $\lim _{n \rightarrow \infty} x_{n}=x$.

Lemma 1.5. For a convergent sequence $\left\{x_{n}\right\}$ in a linear 2normed space $A, \lim _{n \rightarrow \infty}\left\|x_{n}, y\right\|=\left\|\lim _{n \rightarrow \infty} x_{n}, y\right\|$ for all $y \in A$.

Definition 1.6. A linear 2-normed space in which every Cauchy sequence is a convergent sequence is called a 2Banach space.

Throughout this paper, let $A$ be a normed linear space and $B$ be a 2-Banach space. 


\section{STABILITY OF THE QUADRATIC FUNCTIONAL EQUATION (1.1)}

Theorem 2.1. Let $A$ be a linear space, $B$ be a 2-Banach space and $f: A \rightarrow B$ be a mapping with $0 \leq \mu<\infty$ and $0<p<2$ satisfying the inequality

$$
\|f(2 x+y)+f(2 x-y)-f(x+y)-f(x-y)-6 f(x), z\| \leq \mu\left(\|x\|^{p}+\|y\|^{p}\right)
$$

for all $x, y \in A$ and $z \in B$. Then, there exists a unique quadratic mapping $\square: A \rightarrow B$ such that

$$
\|f(x)-\square(x), y\| \leq \frac{4^{(l+1)(m-l)}-2^{l p(m-l)}}{2\left(4-2^{p}\right)} \mu\|x\|^{p}
$$

for all $x \in A$ and $y \in B$.

Proof: Let $y=0$ in $(2.1)$, we get

$$
\begin{aligned}
& \|2 f(2 x)-8 f(x), z\| \leq \mu\|x\|^{p} \\
& \left\|\frac{f(2 x)}{4}-f(x), z\right\| \leq \frac{1}{8} \mu\|x\|^{p}
\end{aligned}
$$

for all $x \in A$ and $z \in B$. Substituting $2 x$ at the place of $x$ and dividing by 4 in the above inequality (2.3), we get

$$
\left\|\frac{f\left(2^{2} x\right)}{4^{2}}-\frac{f(2 x)}{4}, z\right\| \leq \frac{1}{2.4^{2}} \mu\|2 x\|^{p}
$$

for all $x \in A$ and $z \in B$. Again replacing $x$ with $2^{i} x$ and dividing by $4^{i}$ in inequality (2.3), we have $\left\|\frac{f\left(2^{i+1} x\right)}{4^{i+1}}-\frac{f\left(2^{i} x\right)}{4^{i}}, z\right\| \leq \frac{1}{2.4^{i+1}} \mu\left\|2^{i} x\right\|^{p} \leq \frac{1}{2.4^{i+1}} 2^{i p} \mu\|x\|^{p}$

for all $x \in A, z \in B$ and $i \geq 0$. Now, in order to prove that the sequence $\left\{\frac{f\left(2^{n} x\right)}{4^{n}}\right\}$ is a convergent sequence, let us consider $l, m$ be two positive number with $l<m$, such that

$$
\left\|\frac{f\left(2^{m} x\right)}{4^{m}}-\frac{f\left(2^{l} x\right)}{4^{l}}, z\right\| \leq \frac{1}{2} \sum_{i=l}^{m-1} \frac{2^{i p}}{4^{i+1}} \mu\|x\|^{p} \text { (2.6) }
$$

for all $x \in A, z \in B$ and $i \geq 0$. Taking limit on both sides of (2.6), we get

$$
\lim _{m, l \rightarrow \infty}\left\|\frac{f\left(2^{m} x\right)}{4^{m}}-\frac{f\left(2^{l} x\right)}{4^{l}}, z\right\|=0
$$

for all $x \in A$ and all $z \in B$. Which implies that the sequence $\left\{\frac{f\left(2^{n} x\right)}{4^{n}}\right\}$ is a Cauchy sequence in $B$. Since the space $B$ is 2Banach space, the sequence $\left\{\frac{f\left(2^{n} x\right)}{4^{n}}\right\}$ is convergent also. Therefore, we may define a quadratic mapping $\square: A \rightarrow B$ defined by $\square(x)=\lim _{n \rightarrow \infty} \frac{f\left(2^{n} x\right)}{4^{n}}$ for all $x \in A$. Now, to prove that the mapping $\square: A \rightarrow B$ also satisfies the functional equation (1.1), by using Lemma 1.5 and the inequality (2.1), we have

$\|\square(2 x+y)+\square(2 x-y)-\square(x+y)-\square(x-y)-6 \square(x), z\|$

$$
\begin{aligned}
& =\lim _{i \rightarrow \infty} \frac{1}{4^{i}}\left\|f\left(2.2^{i} x+2^{i} y\right)+f\left(2.2^{i} x-2^{i} y\right)-f\left(2^{i} x+2^{i} y\right)-f\left(2^{i} x-2^{i} y\right)-6 f\left(2^{i} x\right), z\right\|^{2} \\
& \leq \lim _{i \rightarrow \infty} \frac{1}{4^{i}} \mu\left(\left\|2^{i} x\right\|^{p}+\left\|2^{i} y\right\|^{p}\right) \leq \lim _{i \rightarrow \infty} \frac{2^{i p}}{4^{i}} \mu\|x\|^{p}+\lim _{i \rightarrow \infty} \frac{2^{i p}}{4^{i}}\|y\|^{p}=0
\end{aligned}
$$

$\|\square(2 x+y)+\square(2 x-y)-\square(x+y)-\square(x-y)-6 \square(x), z\|=0$ for all $x, y \in A$ and all $z \in B$. In order to prove the inequality (2.2) that is the main result of theorem 2.1, by using (2.6), we have

$\|f(x)-\square(x), y\|=\lim _{m \rightarrow \infty}\left\|f(x)-\frac{f\left(2^{m} x\right)}{4^{m}}, y\right\| \leq \frac{4^{(l+1)(m-l)}-2^{l p(m-l)}}{2\left(4-2^{p}\right)} \mu\|x\|^{p}$ for all $x \in A$ and all $y \in B$. Now, to prove the uniqueness of the quadratic mapping $\square: A \rightarrow B$, let us consider another quadratic mapping $\square^{\prime}: A \rightarrow B$ which satisfies the inequality (1.1), we have

$$
\begin{aligned}
& \left\|\square(x)-\square{ }^{\prime}(x), y\right\|=\frac{1}{4^{n}}\left\|\square\left(2^{n} x\right)-\square{ }^{\prime}\left(2^{n} x\right), y\right\| \\
& \leq \frac{1}{4^{n}}\left(\left\|\square\left(2^{n} x\right)-f\left(2^{n} x\right), y\right\|+\left\|\square{ }^{\prime}\left(2^{n} x\right)-f\left(2^{n} x\right), y\right\|\right)
\end{aligned}
$$

which tends to zero as $n \rightarrow \infty$ for all $x \in A$ and all $y \in B$. Hence by Lemma 1.2 we conclude that $\square(x)=\square$ ' $(x)$ for all $x \in A$. Which proves the uniqueness of $\square: A \rightarrow B$. Hence proved the theorem.

Theorem 2.2. Let $A$ be a linear space, $B$ be a 2-Banach space and $f: A \rightarrow B$ be a mapping with $0 \leq \mu<\infty$ and $p>2$ satisfying the inequality

$\|f(2 x+y)+f(2 x-y)-f(x+y)-f(x-y)-6 f(x), z\| \leq \mu\left(\|x\|^{p}+\|y\|^{p}\right)$

for all $x, y \in A$ and $z \in B$. Then, there exists a unique quadratic mapping $\square: A \rightarrow B$ such that

$$
\|f(x)-\square(x), y\| \leq \frac{2^{l p(m-l)}-4^{(l)(m-l)}}{2\left(2^{p}-4\right)} \mu\|x\|^{p}
$$

for all $x \in A$ and $y \in B$.

Proof: Putting $y=0$ in (2.7), we get

$$
\begin{aligned}
& \|2 f(2 x)-8 f(x), z\| \leq \mu\|x\|^{p} \\
& \left\|f(x)-4 f\left(\frac{x}{2}\right), z\right\| \leq \frac{1}{2} \mu\left\|\frac{x}{2}\right\|^{p}
\end{aligned}
$$

for all $x \in A$ and $z \in B$. Substituting $x / 2$ at the place of $x$ and multiplying by 4 in the above inequality (2.9), we get

$$
\left\|4 f\left(\frac{x}{2}\right)-4^{2} f\left(\frac{x}{2^{2}}\right), z\right\| \leq \frac{1}{2} 4 \mu\left\|\frac{x}{2^{2}}\right\|^{p}
$$

for all $x \in A$ and $z \in B$. Again replacing $x$ with $x / 2^{i}$ and multiplying by $4^{i}$ in inequality (2.9), we have

$$
\left\|4^{i} f\left(\frac{x}{2^{i}}\right)-4^{i+1} f\left(\frac{x}{2^{i+1}}\right), z\right\| \leq \frac{1}{2} 4^{i} \mu\left\|\frac{x}{2^{i+1}}\right\|^{p}
$$

for all $x \in A, z \in B$ and $i \geq 0$. Now in order to prove that the sequence $\left\{4^{n} f\left(\frac{x}{2^{n}}\right)\right\}$ is a convergent sequence, let us consider $l, m$ be two positive number with $l<m$, such that 


$$
\left\|4^{m} f\left(\frac{x}{2^{m}}\right)-4^{l} f\left(\frac{x}{2^{l}}\right), z\right\| \leq \frac{\mu}{2} \sum_{i=l}^{m-1} \frac{4^{i}}{2^{i p}}\|x\|^{p}
$$

for all $x \in A, z \in B$ and $i \geq 0$. Taking limit on both sides of (2.10), we get

$$
\lim _{m, l \rightarrow \infty}\left\|4^{m} f\left(\frac{x}{2^{m}}\right)-4^{l} f\left(\frac{x}{2^{l}}\right), z\right\|=0
$$

for all $x \in A$ and all $z \in B$. Which implies that the sequence $\left\{4^{n} f\left(\frac{x}{2^{n}}\right)\right\}$ is a Cauchy sequence in $B$. Since the space $B$ is 2- Banach space, the sequence $\left\{4^{n} f\left(\frac{x}{2^{n}}\right)\right\}$ is convergent also. Therefore, we may define a quadratic mapping $\square: A \rightarrow B$ defined by $\square(x)=\lim _{n \rightarrow \infty} 4^{n} f\left(\frac{x}{2^{n}}\right)$ for all $x \in A$. Further, the remaining proof of this theorem is similar as the proof of Theorem 2.1.

\section{STABILITY OF THE QUADRATIC FUNCTIONAL EQUATION (1.2)}

Theorem 3.1. Let $A$ be a linear space, $B$ be a 2-Banach space and $f: A \rightarrow B$ be a mapping with $0 \leq \mu<\infty$ and $0<p<2$ satisfying the inequality

$\|f(3 x+y)+f(3 x-y)-f(x+y)-f(x-y)-16 f(x), z\| \leq \mu\left(\|x\|^{p}+\|y\|^{p}\right)$

for all $x, y \in A$ and $z \in B$. Then, there exists a unique quadratic mapping $\square: A \rightarrow B$ such that

$$
\|f(x)-\square(x), y\| \leq \frac{9^{(l+1)(m-l)}-3^{l p(m-l)}}{2\left(9-3^{p}\right)} \mu\|x\|^{p}
$$

for all $x \in A$ and $y \in B$.

Proof: Taking $y=0$ in (3.1), we get

$$
\begin{aligned}
& \|2 f(3 x)-18 f(x), z\| \leq \mu\|x\|^{p} \\
& \left\|\frac{f(3 x)}{9}-f(x), z\right\| \leq \frac{1}{18} \mu\|x\|^{p}
\end{aligned}
$$

for all $x \in A$ and $z \in B$. Substituting $3 x$ at the place of $x$ and dividing by 9 in the above inequality (3.3), we get

$$
\left\|\frac{f\left(3^{2} x\right)}{9^{2}}-\frac{f(3 x)}{9}, z\right\| \leq \frac{1}{2.9^{2}} \mu\|3 x\|^{p}
$$

for all $x \in A$ and $z \in B$. Again replacing $x$ with $3^{i} x$ and dividing by $9^{i}$ in inequality (3.3), we have

$$
\left\|\frac{f\left(3^{i+1} x\right)}{9^{i+1}}-\frac{f\left(3^{i} x\right)}{9^{i}}, z\right\| \leq \frac{1}{2.9^{i+1}} \mu\left\|3^{i} x\right\|^{p} \leq \frac{1}{2.9^{i+1}} 3^{i p} \mu\|x\|^{p}
$$

for all $x \in A, z \in B$ and $i \geq 0$. Now in order to prove that the sequence $\left\{\frac{f\left(3^{n} x\right)}{9^{n}}\right\}$ is a convergent sequence, let us consider $l, m$ be two positive number with $l<m$, such that $\left\|\frac{f\left(3^{m} x\right)}{9^{m}}-\frac{f\left(3^{l} x\right)}{9^{l}}, z\right\| \leq \frac{\mu}{2} \sum_{i=l}^{m-1} \frac{3^{i p}}{9^{i+1}} \mu\|x\|^{p}$

for all $x \in A, z \in B$ and $i \geq 0$. Taking limit on both sides of (3.6), we get

$\lim _{m, l \rightarrow \infty}\left\|\frac{f\left(3^{m} x\right)}{9^{m}}-\frac{f\left(3^{l} x\right)}{9^{l}}, z\right\|=0$

for all $x \in A$ and all $z \in B$. Which implies that the sequence $\left\{\frac{f\left(3^{n} x\right)}{9^{n}}\right\}$ is a Cauchy sequence in $B$. Since the space $B$ is 2- Banach space, the sequence $\left\{\frac{f\left(3^{n} x\right)}{9^{n}}\right\}$ is convergent also. Therefore, we may define a quadratic mapping $\square: A \rightarrow B$ defined by $\square(x)=\lim _{n \rightarrow \infty} \frac{f\left(3^{n} x\right)}{9^{n}}$ for all $x \in A$. Now, to prove that the mapping $\square: A \rightarrow B$ also satisfies the functional equation (1.2), by using Lemma 1.5 and the inequality (3.1), we get

$$
\begin{aligned}
& \|\square(3 x+y)+\square(3 x-y)-\square(x+y)-\square(x-y)-16 \square(x), z\| \\
& =\lim _{i \rightarrow \infty} \frac{1}{9^{i}}\left\|f\left(3.3^{i} x+3^{i} y\right)+f\left(3.3^{i} x-3^{i} y\right)-f\left(3^{i} x+3^{i} y\right)-f\left(3^{i} x-3^{i} y\right)-16 f\left(3^{i} x\right), z\right\| \\
& \leq \lim _{i \rightarrow \infty} \frac{1}{9^{i}} \mu\left(\left\|3^{i} x\right\|^{p}+\left\|3^{i} y\right\|^{p}\right) \leq \lim _{i \rightarrow \infty} \frac{3^{i p}}{9^{i}} \mu\|x\|^{p}+\lim _{i \rightarrow \infty} \frac{3^{i p}}{9^{i}}\|y\|^{p}=0
\end{aligned}
$$

$\|\square(3 x+y)+\square(3 x-y)-\square(x+y)-\square(x-y)-16 \square(x), z\|=0$ for all $x, y \in A$ and all $z \in B$. In order to prove the inequality (3.2) that is the main result of theorem 3.1, by using (3.6), we have

$$
\|f(x)-\square(x), y\|=\lim _{m \rightarrow \infty}\left\|f(x)-\frac{f\left(3^{m} x\right)}{9^{m}}, y\right\| \leq \frac{9^{(l+1)(m-l)}-3^{l p(m-l)}}{2\left(9-3^{p}\right)} \mu\|x\|^{p}
$$

for all $x \in A$ and all $y \in B$. Now, to prove the uniqueness of the quadratic mapping $\square: A \rightarrow B$, let us consider another quadratic mapping $\square^{\prime}: A \rightarrow B$ which satisfies the inequality (1.2), we have

$$
\begin{aligned}
& \| \square(x)-\square \text { ' }(x), y\left\|=\frac{1}{9^{n}}\right\| \square\left(3^{n} x\right)-\square \text { ' }\left(3^{n} x\right), y \| \\
& \leq \frac{1}{9^{n}}\left(\left\|\square\left(3^{n} x\right)-f\left(3^{n} x\right), y\right\|+\left\|\square{ }^{\prime}\left(3^{n} x\right)-f\left(3^{n} x\right), y\right\|\right)
\end{aligned}
$$

Which tends to zero as $n \rightarrow \infty$ for all $x \in A$ and all $y \in B$. Hence by Lemma 1.2 we conclude that $\square(x)=\square$ ' $(x)$ for all $x \in A$. Which proves the uniqueness of $\square: A \rightarrow B$. Hence proved the theorem.

Theorem 3.2. Let $A$ be a linear space, $B$ be a 2-Banach space and $f: A \rightarrow B$ be a mapping with $0 \leq \mu<\infty$ and $p>2$ satisfying the inequality

$$
\|f(3 x+y)+f(3 x-y)-f(x+y)-f(x-y)-16 f(x), z\| \leq \mu\left(\|x\|^{p}+\|y\|^{p}\right)
$$

for all $x, y \in A$ and $z \in B$. Then, there exists a unique quadratic mapping $\square: A \rightarrow B$ such that 
$\|f(x)-\square(x), y\| \leq \frac{3^{l p(m-l)}-9^{(l)(m-l)}}{2\left(3^{p}-9\right)} \mu\|x\|^{p}$

for all $x \in A$ and $y \in B$.

\section{CONCLUSION}

First we proved the Hyers-Ulam-Rassias stability of the quadratic functional equations (1.1) in the section 2. Further, in the section 3 we studied the Hyers-Ulam-Rassias stability of the quadratic functional equations (1.2) for the mapping $f$ from orthogonal linear space in to 2-Banach space.

\section{REFERENCES}

[1] A. White, 2-Banach spaces, Doctorial Diss., St. Louis Univ., 1968.

[2] A. White, 2-Banach spaces, Math. Nachr. (42) (1969), pp. $43-60$.

[3] D. H. Hyers, On the stability of the linear functional equation, Proc. Nat. Acad. Sci. (27) (1941), pp. 222-224.

[4] H. A. Kenary, D. Y. Shin, J. R. Lee and H. Hoseini, Fixed Point and Hyers-Ulam stability of functional equations, (5) (37) (2011), pp. 1827-1833.
[5] I. S. Chang and H. M. Kim, On the Hyers-Ulam-Rassias stability of quadratic functional equations, J. Ineq. Pure and Appl. Math., (3) (3) (2002), pp. 1-12.

[6] P. Gavruta, A Generalization of the Hyers-Ulam-Rassias Stability of Approximately Additive Mappings, J. Math. Anal. Appl. (184) (1994), pp. 431-436.

[7] S. G*ahler, 2-metrische R"aume und ihre topologische Struktur, Math. Nachr. (26) (1963), pp. 115-148.

[8] S. G“ahler, Lineare 2-normierte R"aumen, Math. Nachr. (28) (1964), pp. 1-43.

[9] S. G“ahler, Uber “ 2-Banach-R“aume, Math. Nachr. (42) (1969), pp. 335-347.

[10] S. M. Ulam, A Collection of the Mathematical Problems, Interscience Publ., New York, 1960.

[11] Th. M. Rassias, On the stability of the Linear mapping in Banach spaces, Procc. of the Amer. Math. Soc., (72) (2) (1978), pp. 297-300.

[12] Th.M. Rassias, On the Stability of Functional Equations in Banach spaces, J. Math. Anal. Appl., (251) (2000), pp 264-284.

[13] W.-G. Park, Approximate additive mapping in 2-Banach spaces and related topics, J. Math. Anal. Appl. (376) (2011), pp. 193-202. 